\title{
Synthesis and Characterization of PLGA Shell Microcapsules Containing Aqueous Cores Prepared by Internal Phase Separation
}

\author{
Samer R. Abulateefeh ${ }^{1,2}$ and Alaaldin M. Alkilany ${ }^{1}$
}

Received 10 July 2015; accepted 9 September 2015; published online 28 September 2015

\begin{abstract}
The preparation of microcapsules consisting of poly(D,L-lactide-co-glycolide) (PLGA) polymer shell and aqueous core is a clear challenge and hence has been rarely addressed in literature. Herein, aqueous core-PLGA shell microcapsules have been prepared by internal phase separation from acetonewater in oil emulsion. The resulting microcapsules exhibited mean particle size of $1.1 \pm 0.39 \mu \mathrm{m}(\mathrm{PDI}=0.35)$ with spherical surface morphology and internal poly-nuclear core morphology as indicated by scanning electron microscopy (SEM). The incorporation of water molecules into PLGA microcapsules was confirmed by differential scanning calorimetry (DSC). Aqueous core-PLGA shell microcapsules and the corresponding conventional PLGA microspheres were prepared and loaded with risedronate sodium as a model drug. Interestingly, aqueous core-PLGA shell microcapsules illustrated 2.5 -fold increase in drug encapsulation in comparison to the classical PLGA microspheres (i.e., 31.6 vs. 12.7\%), while exhibiting sustained release behavior following diffusion-controlled Higuchi model. The reported method could be extrapolated to encapsulate other water soluble drugs and hydrophilic macromolecules into PLGA microcapsules, which should overcome various drawbacks correlated with conventional PLGA microspheres in terms of drug loading and release.
\end{abstract}

KEY WORDS: internal phase separation; microcapsules; microspheres; poly(D,L-lactide-co-glycolide); risedronate sodium.

\section{INTRODUCTION}

Poly(D,L-lactide-co-glycolide) (PLGA) is a polyester random copolymer consisting of two monomers: lactic acid and glycolic acid. PLGA is a FDA-approved polymer for human use owing to its biocompatibility and biodegradability (1). Therefore, PLGA has been extensively used in biomedical and drug delivery applications (2). Moreover, this polymer is one of the most polymers used successfully in the preparation of microparticles and nanoparticles.

According to the internal structure, PLGA microparticles are classified into two types: microspheres and microcapsules (Fig. 1) (3). Microspheres are monolithic particles characterized with an internal continuous matrix. On the other hand, microcapsules are vesicular particles consist of a polymer shell surrounding a single core (mono-nuclear) or multi-cores (poly-nuclear) filled with oil or water. PLGA microspheres and, to less extent, oily core microcapsules are the most commonly prepared types due to their ease of preparation (4). Unfortunately, both of these types are mainly appropriate for encapsulating hydrophobic drugs; however, they suffer from low encapsulation efficiency of water-soluble drugs, peptides, and proteins (5).

\footnotetext{
${ }^{1}$ Department of Pharmaceutics and Pharmaceutical Technology, Faculty of Pharmacy, The University of Jordan, Amman, 11942, Jordan.

${ }^{2}$ To whom correspondence should be addressed. (e-mail: s.abulateefeh@ju.edu.jo)
}

In fact, fabrication of aqueous core-PLGA shell microcapsules is a clear challenge. Therefore, very limited studies have addressed this point in the literature (6,7). These studies came mainly in an attempt to overcome drawbacks related to the conventional $\mathrm{w}_{1} / \mathrm{o} / \mathrm{w}_{2}$ double emulsion method (6-10). They involved in situ formation of $\mathrm{w}_{1} / \mathrm{o} / \mathrm{w}_{2}$ double emulsion from a single o/w emulsion via spontaneous self-emulsification by using either an appropriate emulsifier such as sodium dioctyl sulfosuccinate (Aerosol OT or AOT) (6) or a copolymer as poly(D,L-lactide)- $b$-poly(2-dimethylaminoethyl methacylate)(PLA- $b$-PDMAEMA) (7).

Internal phase separation is another promising approach proposed firstly by the Vincent group to prepare oil core-shell microcapsules $(4,11-13)$. This approach was then utilized to prepare aqueous core microcapsules using three polymers: poly(tetrahydorfuran), poly(methyl methacrylate), and poly(isobutyl methacrylate) (9). This method involves onestep formation of acetone-water in oil emulsion. The internal phase contains the polymer-forming shell dissolved in a mixture of a volatile good solvent (acetone) and a non-volatile bad solvent (water). After emulsification in oil, gradual evaporation of acetone leads to a decrease in polymer solubility and hence polymer precipitation (coacervation) (11). At optimum balance between the interfacial tensions of different phases (14), polymer migrates to the interface forming a shell surrounding aqueous compartment.

Herein, we employed this method, for the first time, to prepare aqueous core-PLGA shell microcapsules. These 


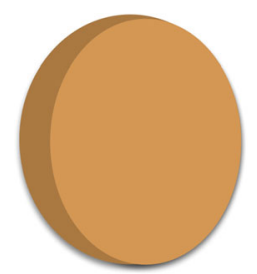

a

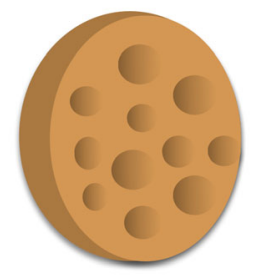

b

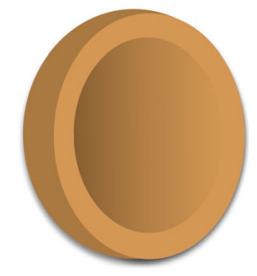

C
Fig. 1. A schematic presentation of internal morphology of a microsphere, b microcapsule with poly-nuclear cores, and $\mathbf{c}$ microcapsule with mono-nuclear core

particles were loaded with risedronate sodium as a model drug. Risedronate is a water-soluble bisphosphonate used in the treatment of osteoporosis (15). Risedronate was used as a model drug because water-soluble drugs are more challenging for incorporation into and release from PLGA particles. As the aim of this manuscript was to fabricate aqueous corePLGA shell microcapsules using an internal phase of (water/ acetone) mixture, the control experiments involved the preparation of the corresponding PLGA microspheres by the same internal phase without water (acetone only). The success of this approach will be utilized for encapsulation of other watersoluble drugs and hydrophilic macromolecules (i.e., peptides and DNA) into PLGA microcapsules, and hence overcome various drawbacks correlated with conventional PLGA microspheres in terms of drug loading and release.

\section{MATERIALS AND METHODS}

\section{Materials}

All chemicals and solvents were used as received without further purification: poly(D,L-lactide-co-glycolide) (PLGA) (50:50) MW ca. 7-17 kDa (Resomer ${ }^{\circledR}$ RG 502 H, Evonik Industries, Germany), sorbitan monooleate $\left(\operatorname{Span}^{\circledR} 80\right.$, Sigma, UK), liquid paraffin (mineral oil, Chemie, Germany), risedronate sodium was a gift from Hikma Pharmaceuticals (Amman, Jordan), acetone 99.5\%, and n-hexane 95\% (Rci Labscan, Thailand).

\section{Preparation of Aqueous Core-PLGA Shell Microcapsules}

Aqueous core-PLGA shell microcapsules were prepared by internal phase separation method adapted from the Vincent group $(9,11-13)$ with modifications. This method involves preparation of two phases: internal acetone-water phase and external oil phase. The former phase was prepared by dissolving PLGA (9 or $30 \mathrm{mg}$ ) in acetone $(3 \mathrm{ml})$ followed by dropwise addition of water $(0.25-0.5 \mathrm{ml})$. The oily phase was prepared by dissolving $\operatorname{Span}^{\circledR} 80$ as emulsifying agent in mineral oil $(12 \mathrm{ml}, 2.5 \% \mathrm{w} / \mathrm{w})$. The two phases were emulsified at $30 \mathrm{~W}$ for $30 \mathrm{~s}$ using ultra-probe sonicator (UP200Ht, Hielscher Ultrasonics, Teltow, Germany). The formed emulsion was left on stirrer until complete evaporation of acetone leading to the formation of hazy suspension. To collect the microcapsules, the resulting suspension was diluted with nhexane $(12 \mathrm{ml})$ and centrifuged at $500 \mathrm{rpm}$ for $5 \mathrm{~min}$ (Centurion Scientific Ltd., Chichester, UK), the precipitated microcapsules were rewashed with n-hexane further two times and left in the fume hood overnight for complete drying. The resulting microcapsules were collected as a dry powder.

Similarly, risedronate-loaded microcapsules were prepared exactly as discussed previously but with dissolving a specific amount of the drug $(6.25 \mathrm{mg})$ in water before addition to the acetone solution.

For comparison purposes, PLGA microspheres (i.e., with no aqueous core) were prepared exactly as mentioned above but without addition of water to the acetone phase. Risedronate-loaded PLGA microspheres were formulated by direct addition of the drug in the acetone phase.

\section{Scanning Electron Microscopy}

Surface morphology of microcapsules was visualized using scanning electron microscopy (SEM; FEI Company-Inspect F50/FEG, the Netherlands). Prior to SEM imaging, dried microcapsules were mounted onto aluminum stubs using doublesided adhesive carbon tape and then coated with gold particles using sputter coater (Quorum Technology, Ashford, UK). Particle size, polydispersity index (PDI), and histograms for particle size distributions were obtained by statistical analysis for a population of particles ( $\mathrm{n} \geq 80$ particles) from SEM micrographs using Image ${ }^{\circledR}$ software.

SEM was also used to examine the internal structure of microspheres and microcapsules. To do so, dried particles were frozen with liquid nitrogen and then fractured by a glass rod before thawing $(9,12,16)$. Fractured particles were prepared for SEM imaging and evaluated as mentioned above.

\section{Differential Scanning Calorimetry and Thermogravimetric Analysis}

Thermal analysis of PLGA polymers, PLGA microspheres, and corresponding aqueous core-PLGA shell microcapsules (with initial amount of polymer $=30 \mathrm{mg}$ ) were conducted using DSC823e Mettler Toledo (Thermo Electron Scientific Instruments Corp., Madison, WI, USA). These samples were placed in aluminum pans and heated at a rate of $10^{\circ} \mathrm{C} /$ min over temperature range between 25 and $250^{\circ} \mathrm{C}$.

Similarly, thermogravimetric analysis (TGA) for these materials was performed using TGA/SDTA 851 Mettler Toledo. These samples $(12-19 \mathrm{mg})$ were placed under nitrogen with flow rate of $10 \mathrm{ml} / \mathrm{min}$ and heated at a rate of $10^{\circ} \mathrm{C} /$ min over temperature range between 25 and $350^{\circ} \mathrm{C}$.

\section{Determination of Drug Loading and Encapsulation Efficiency in Microparticles}

Risedronate loading and encapsulation efficiency from PLGA microparticles (with initial amount of polymer= $30 \mathrm{mg}$ ) were determined by a direct method. A specific amount of dried risedronate-loaded microparticles (25$30 \mathrm{mg}$ ) was dissolved in acetone $(3 \mathrm{ml})$ to confirm liberation of drug molecules from PLGA polymers and then left overnight for complete evaporation. After that, these samples were suspended in phosphate-buffered saline (PBS, $\mathrm{pH}$ 6.8, $3 \mathrm{ml}$ ) and centrifuged at $1500 \mathrm{rpm}$ for $10 \mathrm{~min}$ (Centurion Scientific Ltd., Chichester, UK). The supernatant containing free drug was then collected and analyzed using UV-Vis spectrophotometer (SpectroScan 80D, Biotech Engineering 
Management Co. Ltd., Nicosia, UK) at the corresponding $\lambda_{\text {max }}$ wavelength of $262 \mathrm{~nm}$. The absorbance values were used to determine the amount of risedronate using a calibration curve of risedronate in PBS. Encapsulation efficiency quantification was done in triplicates. Drug encapsulation efficiency and loading were calculated using the following equations (5):

Encapsulation efficiency $\%=\frac{\text { Mass of drug loaded in microparticles }}{\text { Initial mass of drug used in formulation }} \times 100$

Drug loading $\%=\frac{\text { Mass of drug loaded in microparticles }}{\text { Mass ofmicroparticles recovered }} \times 100$

\section{In Vitro Drug Release Studies}

In vitro release studies were conducted for risedronateloaded microspheres and corresponding microcapsules (with initial amount of polymer $=30 \mathrm{mg}$ ) in PBS media. A specific amount of risedronate-loaded microparticles $(25-30 \mathrm{mg})$ was dispersed in PBS ( $\mathrm{pH} 6.8,3 \mathrm{ml}$ ) and placed in a dialysis tube with a molecular weight cutoff $10-12 \mathrm{kDa}$. Dialysis bags were immersed in a glass vial containing PBS ( $\mathrm{pH} 6.8,30 \mathrm{ml}$ ) and placed in a shaker water bath (Heto Lab Equipment, Bromma, Denmark) at $37^{\circ} \mathrm{C}$ and $75 \mathrm{rpm}(12,13,17,18)$. Released amounts of the drug were determined by collecting dialysate samples $(2 \mathrm{ml})$ at certain time intervals and replaced immediately with the same volume of fresh PBS to maintain sink conditions. Collected samples were analyzed using UV-Vis spectrophotometer (SpectroScan 80D, Biotech Engineering Management Co. Ltd., Nicosia, UK) at the corresponding $\lambda_{\max }$ wavelength of $262 \mathrm{~nm}$. Release experiments were done in triplicates, and the average values were recorded with the corresponding standard deviations.

\section{Statistical Analysis}

Data are given as mean \pm standard deviation (SD), $n=3$. Statistical analysis was performed by unpaired $t$ test for drug loading and encapsulation efficiency, and two-way ANOVA with Bonferroni's multiple comparisons test for drug release profiles using GraphPad Prism version 6.0 (San Diego, CA). Results were considered significant when $p<0.05$.

\section{RESULTS}

\section{SEM Characterization}

Internal phase separation method was adapted from Atkin et al. (9) with modifications to prepare aqueous corePLGA shell microcapsules as per cartoon in Fig. 1. For comparison purposes, water-free PLGA microspheres were also prepared by the same method but without adding water to the internal acetone phase. Both surface and internal morphology of particles were characterized by SEM.

PLGA microparticles (with initial amount of polymer= $9 \mathrm{mg}$ ) were characterized by SEM to evaluate surface morphology and size distribution as shown in Fig. 2. Furthermore, particle size, polydispersity index (PDI) and histograms for particle size distribution were obtained by statistical analysis for a population of particles from SEM micrographs using

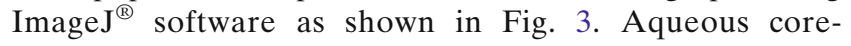
PLGA shell microcapsules exhibited discrete, spherical, and fairly mono-disperse particles with mean particle size of $1.1 \pm 0.39 \mu \mathrm{m}(\mathrm{PDI}=0.35)$ (Figs. 2a and 3a). On the other hand, PLGA microspheres exhibited significantly larger particles with mean particle size of $10.9 \pm 3.1$ $(\mathrm{PDI}=0.29)$ (Figs. 2d and 3b). The significant particle size differences between aqueous core-PLGA shell microcapsules and PLGA microspheres could be attributed to the different composition of the internal phase in each case (acetone/water mixture or acetone, respectively), which dictate the interfacial tension between the internal and the external phases and ultimately the mean droplet size of the resulting water-in-oil emulsion.

The internal morphology of these particles was also revealed by SEM images. For easier evaluation and clearer visualization, larger particles (prepared with initial amount of polymer $=30 \mathrm{mg}$ ) were fractured after freezing in liquid nitrogen (9). Aqueous core-PLGA shell microcapsules exhibited internal poly-nuclear core morphology (Fig. 2b, c). As evident from SEM analysis, a microcapsule with size of $c a$. $15 \mu \mathrm{m}$ contains deep cores with size ranging between 1 and $3 \mu \mathrm{m}$ per core. In addition, many smaller microcapsules exhibited deep central indentation (with a doughnut shape) as shown in Fig. 2c, which would indicate the presence of major empty compartments or holes inside these particles.

In contrast, water-free microspheres exhibited internal continuous monolithic matrices (Fig. 2e) with some spheres having shallow small pores or protrusions (Fig. 2f). Such protrusions are well-reported natural defects happen commonly during solvent evaporation and polymer precipitation (19) and so should be differentiated from the "true" cores observed in microcapsules.

\section{DSC and TGA Analysis}

Thermal analysis of PLGA polymers, aqueous corePLGA shell microcapsules, and the corresponding PLGA microspheres were conducted by DSC as presented in Fig. 4.

The DSC thermogram of PLGA polymer exhibits a sharp endothermic band at $48^{\circ} \mathrm{C}$ (trait a). On the other hand, two characteristic features were observed in the DSC thermogram of the aqueous core-PLGA shell microcapsules (trait b); namely (i) downward shift of the abovementioned endothermic band from 48 to $39^{\circ} \mathrm{C}$ and (ii) emergence of another endothermic band centered at $c a .90^{\circ} \mathrm{C}$. In contrast, only one broad endothermic band centered at $c a .48^{\circ} \mathrm{C}$ is apparent in the DSC thermogram of PLGA microspheres (trait c). Detailed explanation of these thermograms is presented in the Discussion section.

The above samples were also thermally analyzed by thermogravimetric analysis (TGA) (Fig. 5) in an attempt to measure moisture content. However, such measurement was not possible due to similar traits observed for both PLGA microspheres and corresponding aqueous-core PLGA microcapsules, probably due to low detection (sensitivity) and quantification limit of the instrument. 

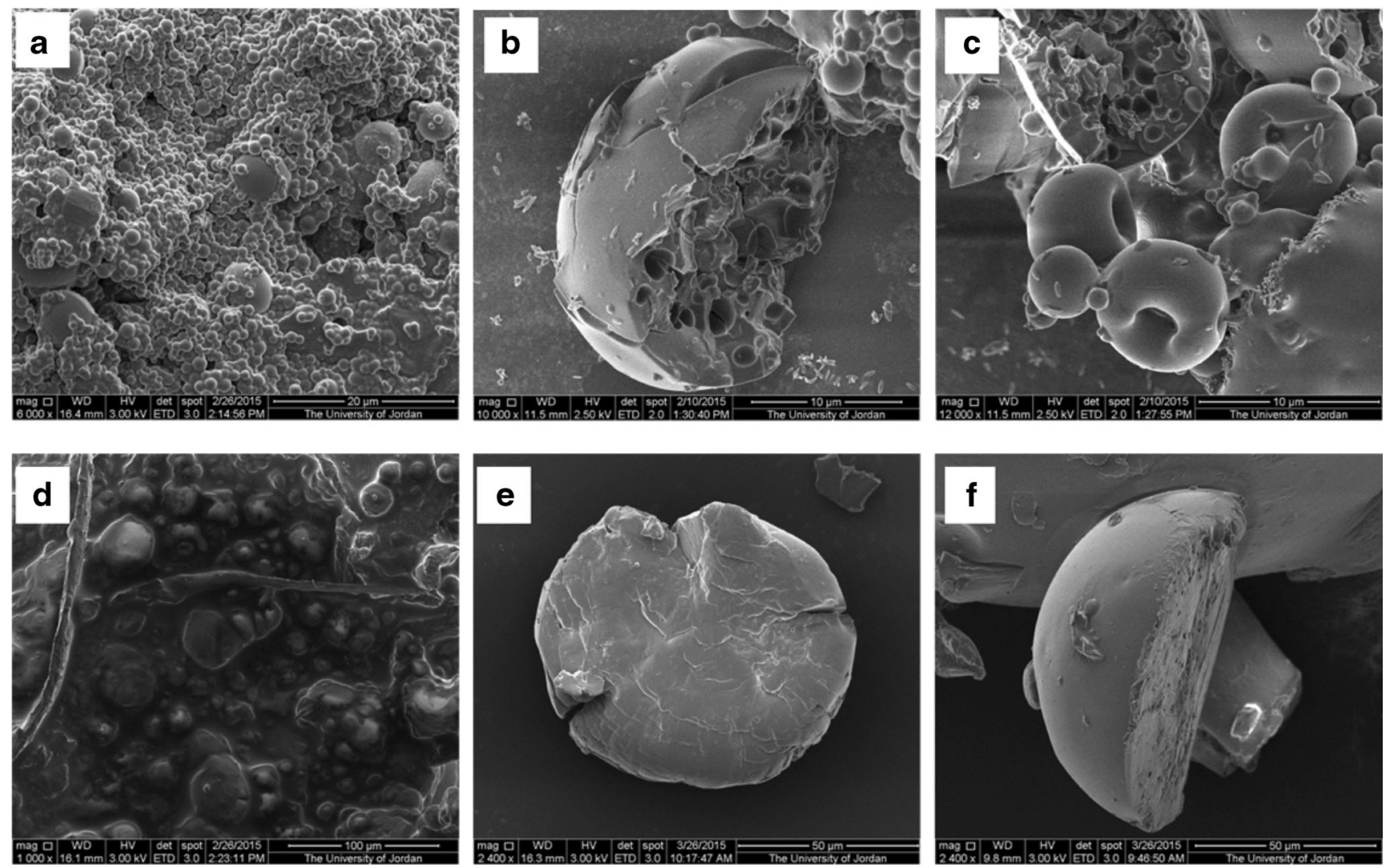

Fig. 2. SEM images of aqueous core-PLGA shell microcapsules (a-c) and PLGA microspheres (d-f). Specifically, the graphs display a a population sample of aqueous core-PLGA shell microcapsules, $\mathbf{b}$ internal morphology of a fractured aqueous core-PLGA shell microcapsule with poly-nuclear core morphology, $\mathbf{c}$ indented aqueous core-PLGA shell microcapsules after fracturing, $\mathbf{d}$ a population of PLGA microspheres, e internal morphology of a fractured PLGA microsphere with continuous monolithic matrix, and $\mathbf{f}$ internal morphology of a fractured PLGA microsphere with defects and shallow pores

\section{Risedronate Loading and Release Studies}

The loaded amount of risedronate drug was measured by a direct method as described in the experimental section. As shown in Fig. 6, PLGA microspheres exhibited drug loading of $3.1 \pm 0.9 \%$ and encapsulation efficiency of $12.7 \pm 4.7 \%$. On the other hand, aqueous core-PLGA shell microcapsules exhibited significant increase ( $c a$. two to threefolds) in drug loading (6.6 \pm $1.2 \%$ ) and encapsulation efficiency (i.e., 31.6 $\pm 5.2 \%$ ).

As shown in Fig. 7, in vitro release studies were conducted for risedronate-loaded microspheres and corresponding microcapsules in $\mathrm{PBS}$ media $\left(\mathrm{pH} 6.8,37^{\circ} \mathrm{C}\right)$. The amount of released drug was measured at specific time points and divided by the total loaded drug (i.e., cumulative drug release \%). Both types of PLGA particles (aqueous core-PLGA shell microcapsules and PLGA microspheres) exhibited similar release profiles. In the first $30 \mathrm{~min}$, PLGA microcapsules and microspheres exhibited burst release of around 49.1 and $43.4 \%$ of their drug content, respectively. However, both types of microparticles exhibited extended release profiles within the remaining $6 \mathrm{~h}$.
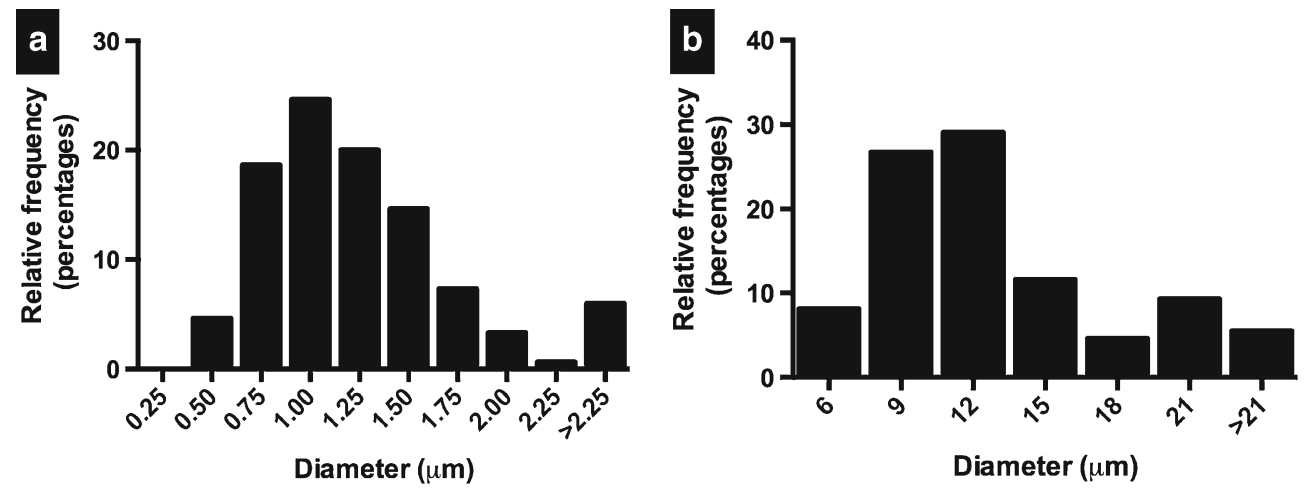

Fig. 3. Histograms for particles size distribution of a aqueous-core PLGA shell microcapsules and b PLGA microspheres 


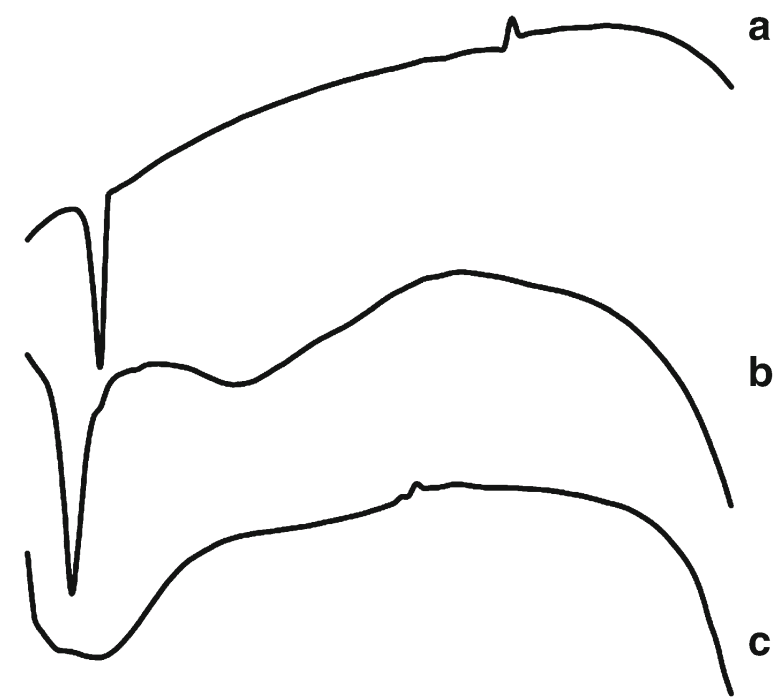

Temperature $\left({ }^{\circ} \mathrm{C}\right)$

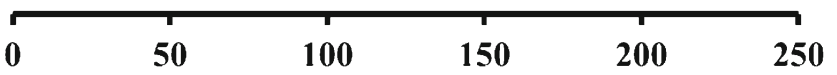

Fig. 4. The DSC thermograms of $a$ PLGA polymers, $b$ aqueous core-PLGA shell microcapsules, and $c$ PLGA microspheres

\section{DISCUSSION}

The preparation of aqueous core-PLGA shell microcapsules is a clear challenge, albeit it would overcome many drawbacks correlated with the conventional PLGA microspheres in terms of drug loading and release, especially for water-soluble drugs and hydrophilic macromolecules. In this work, we employed internal phase separation approach $(9,11)$ with modifications to prepare aqueous core-PLGA shell microcapsules. PLGA polymers were dissolved in a mixture of acetone (volatile good solvent) and water (non-volatile bad solvent) and then emulsified into mineral oil external phase containing Span ${ }^{\circledR} 80$ as emulsifier. Upon acetone evaporation

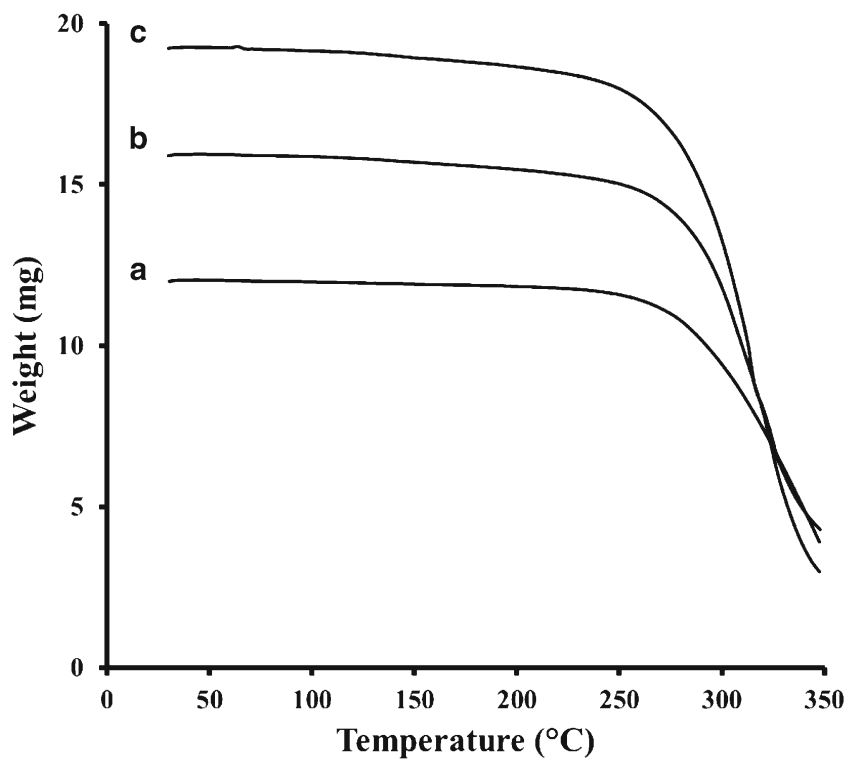

Fig. 5. The TGA thermograms of $a$ PLGA polymers, $b$ aqueous core-PLGA shell microcapsules, and $c$ PLGA microspheres
IIII. Aqueous core-PLGA shell microcapsules - PLGA microspheres

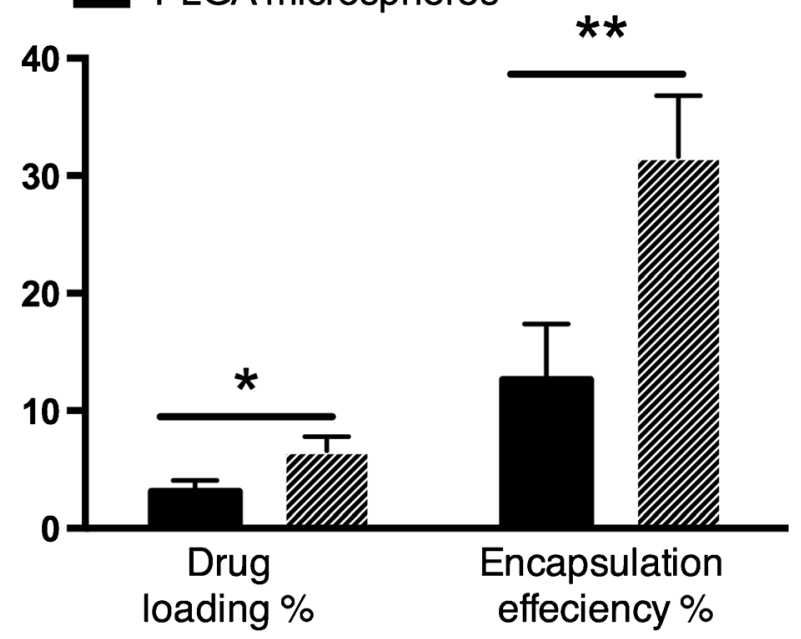

Fig. 6. Drug loading and encapsulation efficiency of risedronate into aqueous core-PLGA shell microcapsules and PLGA microspheres. Error bars represent the standard deviation of three measurements. Data are given as mean \pm standard deviation $(S D) ; n=3$ for each group; unpaired $t$ test; $* p<0.05$ and $* * p<0.01$

from the internal phase droplets, the solubility of PLGA polymers decreases gradually leading to phase separation and migration to the interface surrounding water droplets.

In the internal acetone-water phase, the minimum volume (acetone to water) ratio required to dissolve (30 $\mathrm{mg}$ ) PLGA was 6:1. Interestingly, this ratio is significantly lower than that used in previous studies required to prepare microcapsules using other polymers as poly(tetrahydorfuran) (12:1) and poly(methyl methacrylate) (20:1) (9). Such differences in acetone-to-water ratio would be attributed to the differences of the intrinsic solubility of these polymers into acetone-water mixture. In PLGA case, low acetone-to-water ratio indicates the ability to incorporate larger amounts of water into microcapsules with lower amounts of organic solvents and, thus overcoming one of the major drawbacks correlated with the internal phase separation method used in the preparation of microcapsules composed of poly(tetrahydorfuran),

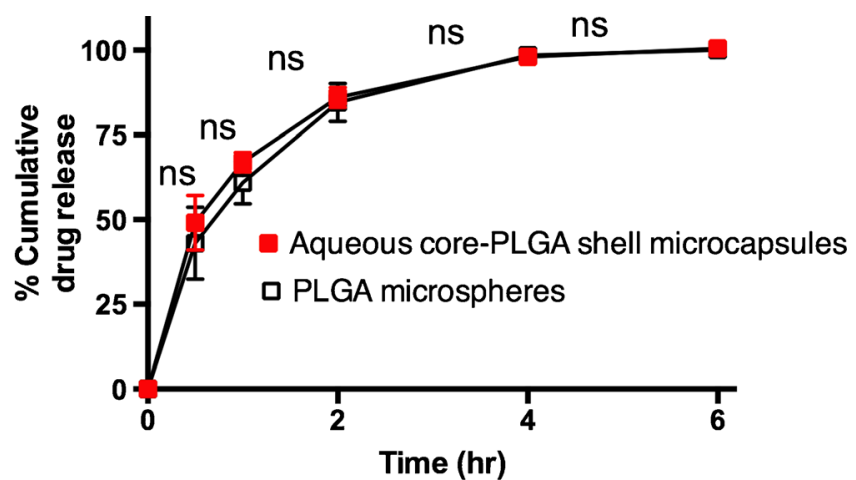

Fig. 7. \% Cumulative release of risedronate from aqueous core-PLGA shell microcapsules and PLGA microspheres in PBS (pH 6.8). Data are given as mean \pm standard deviation $(S D) ; n=3$ for each time point in both curves; $n s$ represents no significant difference at $p<0.05$ using two-way ANOVA with Bonferroni's multiple comparisons test 
poly(methyl methacrylate), and poly(isobutyl methacrylate) polymers $(10,20)$.

The incorporation of water molecules into aqueous corePLGA shell microcapsule were confirmed by SEM images (Fig. 2) and DSC thermal analysis (Fig. 4).

In contrast to the internal continuous matrix of water-free PLGA microspheres (Fig. 2d-f), SEM images revealed the poly-nuclear nature of aqueous core microcapsules (Fig. 2a-c). The formation of multi-cores (poly-nuclear) rather than a single core (mono-nuclear) is probably attributed to two reasons: (i) the rigid nature of PLGA polymers (i.e., glass transition temperature $\left(T_{\mathrm{g}}\right)$ is around $48^{\circ} \mathrm{C}$ ) would slow down its migration to the periphery during acetone evaporation, leading to local precipitation around water droplets entrapped into several cores. Similar findings were observed in previous studies using polymers with comparable $T_{\mathrm{g}}$ values (i.e., poly(methyl methacrylate) and poly(isobutyl methacrylate) polymers). (ii) The volume of acetone used here was just enough to dissolve PLGA polymers in the presence of water molecules. Hence, the evaporation of the first parts of acetone induces fast local precipitation of PLGA polymers with no time to allow polymer migration to the interface (9).

DSC thermal analysis was also utilized to confirm the incorporation of water into microcapsules (Fig. 4). The DSC thermogram of PLGA polymer exhibits a typical sharp endothermic band at $48^{\circ} \mathrm{C}$ indicative of the relaxation peak following the glass transition temperature $\left(T_{\mathrm{g}}\right)$ of this polymer (trait a) (21). Interestingly, the DSC thermogram of the corresponding aqueous core-PLGA shell microcapsules exhibits downward shift of this endothermic band to $\mathrm{ca} .39^{\circ} \mathrm{C}$ as shown in trait b. Such downward shift resulted mainly from the presence of water molecules, which acts as a plasticizer leading to a decrease in the $T_{\mathrm{g}}$ (21-23). Moreover, the incorporation of water molecules into these microparticles was also confirmed by the emergence of another endothermic band centered at $\mathrm{ca} .90^{\circ} \mathrm{C}$ relevant to the evaporation of water molecules $(17,21,24,25)$. On the other hand, this evaporation band is absent in the DSC thermogram of PLGA microspheres with only one broad endothermic band centered at $c a .48^{\circ} \mathrm{C}$ related to the $T_{\mathrm{g}}$ of the polymer (trait c). In contrast to the sharp endothermic $T_{\mathrm{g}}$-related bands observed in the DSC thermograms of PLGA polymers and microcapsules, the broadness of $T_{\mathrm{g}}$ band for microspheres is presumably attributed to the heterogeneous exposure of PLGA polymers to heat (i.e., embedded into matrices or exposed at surface).

Both types of PLGA microparticles were loaded with risedronate sodium model drug and compared in terms of drug loading (Fig. 6) and release profiles (Fig. 7). Risedronate sodium is a water-soluble drug, so it was chosen here as a model drug because it is more challenging for drug incorporation and release into hydrophobic PLGA particles. In fact, PLGA particles prepared by conventional methods such as emulsion evaporation and interfacial deposition methods are more appropriate for water-insoluble drugs but suffer from low encapsulation of water-soluble drugs (5). Hence, the presented protocol shows an alternative simple approach to prepare PLGA particles with higher incorporation of water soluble drugs via core-shell structure.

As shown in Fig. 6, the encapsulation efficiency of watersoluble risedronate sodium was significantly higher in microcapsules as compared to that in microspheres (2.5-folds), which is in agreement with the formation of internal aqueous cores inside the hydrophobic PLGA matrix. The presence of water in the internal phase enhances drug retention into microcapsules and reduces leaching of the drug out to the external phase during preparation leading to higher incorporation.

In vitro drug release studies showed similar release profiles for both microspheres and microcapsules (Fig. 7). Both types exhibited burst release of around 49.1 and $43.4 \%$ of their drug content, respectively. Burst release results from fast diffusion of drug molecules either adsorbed at or entrapped near the surface of particles $(26,27)$. Future studies will be performed to reduce burst drug release including increasing concentrations of PLGA polymers, varying ratios between PLGA polymer to risedronate drug, varying concentrations of emulsifier, and varying ratios between water and oil. However, both types of microparticles exhibited extended release profiles within the remaining $6 \mathrm{~h}$. Kinetics of drug release profiles were analyzed using the Higuchi model $(28,29)$ by employing Eq. 3 :

$$
\frac{M_{t}}{M_{\infty}}=k \sqrt{t}
$$

where $M_{t}$ and $M_{\infty}$ refer to the cumulative amount of drug release at time $t$ and infinite time, respectively, and $k$ is the rate constant.

Drug release from both microspheres and microcapsules were fitted into the Higuchi model, with $R^{2}$ values of 0.99 and 0.97 , respectively. Analysis of drug release profiles for both types suggests Fickian diffusion-controlled release mechanism (28-30). Such non-zero release kinetics (case I transport mechanism) was expected for microspheres and microcapsules with poly-nuclear core morphology. In the latter particles, the rate of drug diffusion is probably limited by diffusion through the matrix rather than that from aqueous reservoir. Therefore, if zero-order release kinetics is desired, mono-nuclear microcapsules with single aqueous core and saturated drug content should be considered (3), which is an ongoing research in our laboratory. Furthermore, systematic optimization studies are in process to investigate the effect of many other factors such as polymer concentration and water to oil ratios.

\section{CONCLUSION}

Internal phase separation approach was employed successfully to prepare aqueous core-PLGA shell microcapsules. DSC and SEM analysis confirmed the presence of internal aqueous poly-nuclear core. These microcapsules were spherical in shape and fairly mono-disperse in size with mean particle size of $1.1 \pm 0.39 \mu \mathrm{m}(\mathrm{PDI}=0.35)$. In comparison to conventional PLGA microspheres, aqueous core-PLGA shell microcapsules exhibited 2.5-fold increase in drug encapsulation efficiency. Systematic optimization studies are in process to prepare aqueous core-PLGA shell microcapsules with mononuclear core morphology.

\section{ACKNOWLEDGMENTS}

The authors thank the Deanship of Academic Research (DAR) at The University of Jordan for the financial support. The authors also acknowledge the technical help of Miss Fatema Abdullah and Miss Sundus Alfarasin. 


\section{REFERENCES}

1. Makadia HK, Siegel SJ. Poly lactic-co-glycolic acid (PLGA) as biodegradable controlled drug delivery carrier. Polymers. 2011;3(3):1377-97.

2. Anderson JM, Shive MS. Biodegradation and biocompatibility of PLA and PLGA microspheres. Adv Drug Deliv Rev. 2012;64(Supplement(0)):72-82.

3. Andersson Trojer M, Nordstierna L, Nordin M, Nyden M, Holmberg K. Encapsulation of actives for sustained release. Phys Chem Chem Phys. 2013;15(41):17727-41.

4. Romero-Cano MS, Vincent B. Controlled release of 4nitroanisole from poly(lactic acid) nanoparticles. J Control Release. 2002;82(1):127-35.

5. Govender T, Stolnik S, Garnett MC, Illum L, Davis SS. PLGA nanoparticles prepared by nanoprecipitation: drug loading and release studies of a water soluble drug. J Control Release. 1999;57(2):171-85.

6. Yu X, Zhao Z, Nie W, Deng R, Liu S, Liang R, et al. Biodegradable polymer microcapsules fabrication through a template-free approach. Langmuir. 2011;27(16):10265-73.

7. Watanabe T, Kimura Y, Ono T. Monodisperse polylactide microcapsules with a single aqueous core prepared via spontaneous emulsification and solvent diffusion. RSC Adv. 2014;4(10):4872-7.

8. Tewes F, Munnier E, Antoon B, Ngaboni Okassa L, CohenJonathan S, Marchais $\mathrm{H}$, et al. Comparative study of doxorubicin-loaded poly(lactide-co-glycolide) nanoparticles prepared by single and double emulsion methods. Eur J Pharm Biopharm. 2007;66(3):488-92.

9. Atkin R, Davies P, Hardy J, Vincent B. Preparation of aqueous core/polymer shell microcapsules by internal phase separation. Macromolecules. 2004;37(21):7979-85.

10. Park JM. Polymeric Hollow Particles for Encapsulation of Chemical Molecules. Encapsulation Nanotechnologies: John Wiley \& Sons, Inc.; 2013. p. 291-345.

11. Loxley A, Vincent B. Preparation of poly(methylmethacrylate) microcapsules with liquid cores. J Colloid Interface Sci. 1998;208(1):49-62.

12. Dowding PJ, Atkin R, Vincent B, Bouillot P. Oil core-polymer shell microcapsules prepared by internal phase separation from emulsion droplets. I. Characterization and release rates for microcapsules with polystyrene shells. Langmuir. 2004;20(26):11374-9.

13. Dowding PJ, Atkin R, Vincent B, Bouillot P. Oil core/polymer shell microcapsules by internal phase separation from emulsion droplets. II: controlling the release profile of active molecules. Langmuir. 2005;21(12):5278-84.

14. Torza S, Mason SG. Three-phase interactions in shear and electrical fields. J Colloid Interface Sci. 1970;33(1):67-83.

15. Nasr M, Awad GAS, Mansour S, Al Shamy A, Mortada ND. A reliable predictive factorial model for entrapment optimization of a sodium bisphosphonate into biodegradable microspheres. J Pharm Sci. 2011;100(2):612-21.

16. Jiang Y, Wang D, Zhao T. Preparation, characterization, and prominent thermal stability of phase-change microcapsules with phenolic resin shell and n-hexadecane core. J Appl Polym Sci. 2007;104(5):2799-806.

17. Abulateefeh SR, Taha MO. Enhanced drug encapsulation and extended release profiles of calcium-alginate nanoparticles by using tannic acid as a bridging cross-linking agent. J Microencapsul. 2015;32(1):96-105.

18. Gun WJ, Routh AF. Formation and characterization of $\mathrm{pH}$-responsive liquid core microcapsules. Langmuir. 2013;29(40):12541-8.

19. Mao S, Shi Y, Li L, Xu J, Schaper A, Kissel T. Effects of process and formulation parameters on characteristics and internal morphology of poly(d, l-lactide-co-glycolide) microspheres formed by the solvent evaporation method. Eur J Pharm Biopharm. 2008;68(2):214-23.

20. Yow HN, Routh AF. Formation of liquid core-polymer shell microcapsules. Soft Matter. 2006;2(11):940-9.

21. Passerini N, Craig DQM. An investigation into the effects of residual water on the glass transition temperature of polylactide microspheres using modulated temperature DSC. J Control Release. 2001;73(1):111-5.

22. Levine H, Slade L. Water as a plasticizer: physico-chemical aspects of low-moisture polymeric systems. In: Franks F, editor. Water science reviews. Cambridge: Cambridge University Press; 1988. p. 79-185.

23. Blasi P, D'Souza SS, Selmin F, DeLuca PP. Plasticizing effect of water on poly(lactide-co-glycolide). J Control Release. 2005;108(1):1-9.

24. AlKhatib HS, Taha MO, Aiedeh KM, Bustanji Y, Sweileh B. Synthesis and in vitro behavior of iron-crosslinked N-methyl and N-benzyl hydroxamated derivatives of alginic acid as controlled release carriers. Eur Polym J. 2006;42(10):2464-74.

25. Taha MO, Nasser W, Ardakani A, AlKhatib HS. Sodium lauryl sulfate impedes drug release from zinc-crosslinked alginate beads: switching from enteric coating release into biphasic profiles. Int J Pharm. 2008;350(1-2):291-300.

26. Washington C. Drug release from microdisperse systems: a critical review. Int J Pharm. 1990;58(1):1-12.

27. Redhead HM, Davis SS, Illum L. Drug delivery in poly(lactideco-glycolide) nanoparticles surface modified with poloxamer 407 and poloxamine 908: in vitro characterisation and in vivo evaluation. J Control Release. 2001;70(3):353-63.

28. Higuchi T. Rate of release of medicaments from ointment bases containing drugs in suspension. J Pharm Sci. 1961;50(10):874-5.

29. Higuchi T. Mechanism of sustained-action medication. Theoretical analysis of rate of release of solid drugs dispersed in solid matrices. J Pharm Sci. 1963;52(12):1145-9.

30. Peppas NA. Analysis of Fickian and non-Fickian drug release from polymers. Pharm Acta Helv. 1984;60(4):110-1. 